\title{
La comunicación de los portales de descuentos y cupones a través de Facebook
}

\author{
Araceli Castelló Martínez \\ Departamento de Comunicación y Psicología Social \\ Universidad de Alicante
}

\section{Palabras clave}

Marketing, comunicación, publicidad, comercio electrónico, redes sociales

\section{Resumen}

El crecimiento experimentado por el comercio electrónico y las plataformas 2.0 ha venido acompañado por el surgimiento y desarrollo de portales caracterizados por ofrecer a los usuarios la compra de productos o contratación de servicios con llamativos descuentos o promociones. El objetivo de este artículo se centra en el estudio de la comunicación que llevan a cabo los llamados portales de couponing a través de sus espacios en la red social Facebook. Se parte de la hipótesis de que estos portales se limitan a volcar la información sobre las ofertas, descuentos y promociones en la red social y generan poca conversación con sus seguidores, a pesar del popular seguimiento que tienen entre los usuarios. La metodología se basa, por una parte, en la investigación documental de publicaciones de los ámbitos profesional y académico, repasando la situación actual de los medios sociales, revisando la evolución vivida por el comercio electrónico y estudiando las particularidades del social commerce, y en el análisis de case studies de los principales portales de descuentos y cupones, por otra. Tras este análisis, queda demostrado el principal uso que los portales de couponing hacen de su presencia en Facebook, como mero canal de difusión y generación de tráfico hacia las ofertas y descuentos, limitando a ello el uso de este entorno colaborativo y ofreciendo escasa conversación con el usuario. 


\title{
Discounts and couponing sites communications through Facebook
}

\section{Keywords}

Marketing, communication, advertisting, e-commerce, social networks

\begin{abstract}
Electronic commerce and 2.0 platforms growth has been accompanied by the emergence and development of web sites which are notable for offering users products and services with attractive discounts or promotions. The aim of this article focuses on studying couponing sites communication through their spaces in the social network Facebook. It starts from the hypothesis that these web sites only use their spaces in Facebook to publish information about offers, discounts and promotions and generate limited conversation with their followers, despite popular follow-up they have. The method is based on documentary research of both, academic and professional publications, reviewing current situation of social media, revising development experienced by e-commerce and studying peculiarities of social commerce, on the one hand, and the analysis of case studies of major couponing and discounts sites, on the other. After this analysis, it is demonstrated that couponing sites use their presence in Facebook mainly as mere diffusion and traffic generation to offers and discounts channel, reducing their communications in this collaborative environment to this goal and offering limited conversation with user.
\end{abstract}

\begin{abstract}
Autora
Araceli Castelló Martínez [Araceli.castello@ua.es] es profesora en la Universidad de Alicante en la asignatura Introducción a la investigación de medios y colaboradora en diversos postgrados. Es doctora en Comunicación, magíster en Comunicación Integral y licenciada en Publicidad y RR.PP. Ha publicado el libro Estrategias empresariales en la Web 2.0, así como informes, artículos y capítulos sobre los medios sociales y la comunicación corporativa y publicitaria online.
\end{abstract}




\section{Introducción}

La aparición del medio Internet y el posterior uso intensivo de las Tecnologías de la Información y la Comunicación (TIC) han supuesto cambios en la concepción general de la comunicación, sus elementos constitutivos y modelos explicativos $^{1}$. Debido a las posibilidades de conectividad entre los individuos, para ser eficaz y cumplir los objetivos previamente definidos la comunicación digital ha de ser participativa e interactiva, con el fin de generar y aportar valor añadido al cliente actual y/o potencial y crear vínculos emocionales entre la marca/empresa y los usuarios.

La red es hoy en día uno de los canales fundamentales en la interacción y la comunicación de personas, empresas e instituciones. De acuerdo con la segunda oleada de 2011 del Estudio General de Medios publicado por la Asociación para la Investigación de Medios de Comunicación (AIMC)², la audiencia mensual de Internet asciende al $57,4 \%$ de la población española de 14 años o más y el $75 \%$ de los internautas accede a la red a diario.

La inversión publicitaria en medios interactivos ha vivido en los últimos años un crecimiento muy rápido: Internet $(13,5 \%)$ es el tercer medio en el reparto de la inversión publicitaria en medios convencionales en España, según el Estudio InfoAdex de la inversión publicitaria en España 2011 publicado por InfoAdex ${ }^{3}$ con 789,54 millones de euros, y de enero a septiembre de 2011 la inversión publicitaria en Internet creció un 8,5\% con respecto a los nueve primeros meses de $2010^{4}$.

Internet se consolida así como el medio más consumido por los españoles, de tal manera que la industria y el sector publicitario son hoy más online que nunca, como señala Madinabeitia (2010: 43) al afirmar que «la digitalización de la sociedad ha traído consigo grandes cambios en todos los ámbitos, siendo el mundo de la comunicación y en concreto el de la comunicación comercial uno de los más afectados». Las principales ventajas que hacen del medio online un canal cuanto menos necesario en los planes estratégicos de los anunciantes, son:

- Alta capacidad de afinidad y microsegmentación.

- Amplia cobertura, por su elevada penetración en la población.

- Interactividad: comunicación de ida y vuelta con usuarios, clientes actuales o potenciales.

- Evaluación y optimización del retorno de la inversión (ROI) en tiempo real y exhaustiva medición post-campaña.

\footnotetext{
1 Bryant y Miron (2004: 697) concluyen en su estudio que ninguna de las teorías de la comunicación de masas más populares del siglo XX parece "particularmente preparada para explicar, predecir o ni siquiera servir a los grandes cambios que se están produciendo en nuestras instituciones mediáticas, en el sistema de mensajes y audiencias».

2 Puede consultarse en www.aimc.es. Fecha de consulta 19/11/2011.

3 Disponible en www.infoadex.es. Fecha de consulta 19/11/2011.

4 http://www.optimediaintelligence.es/noticias_archivos/458_20111025170411.pdf. Fecha de consulta 19/11/2011.
} 
- Acceso a un potencial consumidor muy cualificado, exigente y proactivo (prosumer) gracias a toda la información que tiene a su alcance en el espacio digital, y que cada vez más practica el multitasking ${ }^{5}$.

- Generación de notoriedad, recuerdo y branding con costes reducidos.

- Canal de venta y fidelización.

- Creación de comunidades en torno a la marca (engagement).

- Complementariedad con otros medios, demostrada por los estudios cross media.

- Flexibilidad y amplia variedad de formatos, soportes, modelos de negocio, etc.

La explosión de plataformas en Internet al servicio del marketing y la comunicación empresarial, derivadas de los espacios de la Web 2.0 y fundamentalmente basadas en la integración de contenidos, ha hecho que entornos colaborativos como Facebook y Twitter también se empleen como un nuevo canal de venta y atención al cliente, teniendo en cuenta el papel que juega la influencia social en el comportamiento de compra y consumo del usuario así como el espacio de conversación, sobre temáticas como las marcas, que representan hoy en día las redes sociales.

El consumidor social cada vez toma más decisiones de compra y consumo basándose en opiniones, experiencias y comentarios que comparte con otros usuarios en entornos colaborativos como Facebook y busca en estas plataformas descuentos, promociones y ofertas. Este fenómeno ha supuesto una redefinición de la manera en que los individuos interactúan con marcas y empresas.

Dentro del llamado social commerce, las plataformas online de compras colectivas han adquirido entre 2010 y 2011 gran protagonismo en el panorama digital, en general, y los medios sociales, en particular. En este artículo estudiaremos la comunicación llevada a cabo por los portales de descuentos y cupones a través de sus espacios en Facebook, como canales a través de los cuales los usuarios pueden compartir sus experiencias y socializar, más si cabe, el proceso de compra y consumo.

\section{Metodología}

El objetivo de este artículo se centra en el estudio de la comunicación que llevan a cabo los llamados portales de couponing a través de sus espacios en la red social Facebook, con el fin de analizar la tipología de contenidos publicados y la interacción con los seguidores. Se parte de la hipótesis de que estos portales se

5 Según el estudio «Los multitaskers europeos« de Microsoft Corporation, el 56\% de los internautas navegan por Internet mientras ven la televisión. 
limitan a volcar la información sobre las ofertas, descuentos y promociones en la red social y generan poca conversación con sus seguidores, a pesar del popular seguimiento que tienen entre los usuarios.

La metodología se basa, por una parte, en la investigación documental de publicaciones de los ámbitos profesional y académico relacionadas con la temática del artículo, repasando en el marco teórico la situación actual de los medios sociales, revisando la evolución vivida por el comercio electrónico y estudiando las particularidades del social commerce, y en el análisis de case studies de los principales portales de descuentos y cupones, por otra.

La localización de información ya publicada sobre el tema de estudio permite establecer un marco de referencia del fenómeno al analizar los estudios e investigaciones realizados sobre la materia en los últimos cinco años, dada la actualidad de la temática, el uso de las plataformas 2.0 como canal de comunicación, venta y atención al cliente.

Para localizar publicaciones desde el ámbito académico se emplean los buscadores Dialnet (www.dialnet.org) y Google Academics (http://scholar.google.es/). La identificación de estudios y publicaciones desde el ámbito profesional se realiza mediante la búsqueda en Google Books (http://books.google.es) así como en los ocho diarios digitales de marketing, comunicación y publicidad en español más importantes en volumen de audiencia ${ }^{6}$. En ambos casos se emplean una serie de palabras clave relacionadas con el tema de estudio para la identificación de estudios y publicaciones: comunicación, marketing, publicidad, social media, redes sociales, Internet, comercio electrónico, social commerce, fidelización, Facebook, Twitter, venta online y Return On Investment (ROI).

Con respecto a los case studies, la selección de portales de cupones y descuentos se basa en el ranking de la empresa de medición Nielsen publicado en julio de 2011 para esta categoría, y liderado por las empresas Groupon (con 2,5 millones de usuarios), Offerbox (con un tráfico de 2 millones de audiencia), LetsBonus y Groupalia, con 1,3 millones de usuarios cada una de ellas ${ }^{7}$. El listado de espacios analizados lo completan los portales Planeo, Mequedouno, Oooferton, Oportunista, Offerum, Quebombazo, Cuponing y Cupooon. Estos doce portales de couponing se encuentran además, entre los primeros resultados de las búsquedas de las palabras «cupones» $\mathrm{y}$ «descuentos» en Google, contando la mayoría de ellos con enlaces patrocinados en la parte superior y la columna derecha de los resultados de búsqueda ${ }^{8}$.

6 De acuerdo con las rankings de ComScore y Nielsen, éstos son: www.puromarketing.com, www.marketingdirecto. com, www.marketingnews.es, www.interactivadigital.com, www.mediosymarketing.es, www.ipmark.com, www.elpublicista.es y www.brandlife.es.

7 Puede consultarse en: http://www.puromarketing.com/53/10434/groupon-offerbox-letsbonus-lideran-portalesdescuentos-cuponing.html

8 Búsqueda realizada el día 14/11/2001. 
Puesto que una de las peculiaridades de las promociones y descuentos ofrecidos por este tipo de portales es su carácter local, se analiza la comunicación en sus espacios de Facebook en el ámbito de la provincia de Alicante, en caso de que así exista, ya que la mayoría de estas empresas cuentan con presencia específica en la red social para cada uno de sus ámbitos de actuación.

Para cada uno de estos case studies, los ítems analizados entre el 10 y el 20 de noviembre de 2011 son los siguientes:

- dirección de página web y página en Facebook.

- traffic rank en el medidor de tráfico gratuito Alexa en octubre de 2011 y número de fans en Facebook.

- número de ofertas publicadas en la página web y número de ofertas publicadas en Facebook, con la media diaria.

- tipología de publicaciones en la página de Facebook: promociones y/o descuentos, información complementaria y/o noticias relacionadas, encuestas $\mathrm{y} / \mathrm{o}$ preguntas a usuarios u otros.

- resultados de las publicaciones en Facebook: número de «personas que están hablando de esto», número de «me gusta», número de publicaciones compartidas por usuarios y número de comentarios de usuarios ${ }^{9}$.

- respuestas a comentarios y dudas de usuarios, en caso de que existan.

- otros contenidos de la página.

A partir del análisis de los resultados para cada uno de estos ítems y de la investigación documental del marco teórico se extraen las conclusiones para verificar o refutar la hipótesis inicial, basada en la escasa conversación generada por los portales de cupones y descuentos con sus seguidores en la plataforma Facebook.

\section{Marco teórico}

En este apartado contextualizaremos el fenómeno de estudio a partir de la información obtenida en la investigación documental y a través de diferentes puntos: la Web 2.0 y los medios sociales, el comercio electrónico, el social commerce y los portales de descuentos y cupones.

\subsection{La Web 2.0 y los medios sociales}

El receptor se ha convertido en el eje de los procesos comunicativos de la empresa a través de plataformas digitales, dadas sus capacidades de participación e interacción. La Web 2.0 alude a la red como un espacio social y se enmarca

9 Distinguimos entre los comentarios publicados por usuarios en las páginas en Facebook de los portales analizados y las publicaciones de la página que han sido compartidas por usuarios. 
dentro de la teoría de la comunicación digital interactiva, caracterizada por las hipermediaciones (Scolari, 2008: 113-114).

Espacios como los blogs o las redes sociales han permitido al usuario pasar de ser mero consumidor pasivo de contenidos a generarlos (User Generated Content), editarlos según sus intereses y compartirlos con su comunidad: «el receptor además de audiencia, de público y de consumidor, deviene usuario y prosumer, es decir, co-productor, distribuidor y consumidor, todo al mismo tiempo» (Bermejo, 2008: 49).

Términos como crossumer, prosumer, fansumer o persumer destacan la «dimensión interactiva del usuario» (Marshall, 2004: 51), que ha modificado el concepto de audiencia en entornos colaborativos y tecnologías participativas.

Desde el lado de la empresa, escuchar y relacionarse con los clientes a través de las comunidades de la marca en estos nuevos espacios, buscando generar el engagement, supone una transformación total de la cultura y la comunicación corporativas, al integrar precisamente en el centro del proceso al consumidor, actual y/o potencial, según la filosofía 2.0. Afirma Martí Parreño (2006: 6) que:

\begin{abstract}
«los mensajes están siendo sustituidos por 'contextos de comunicación' en torno a las marcas con los que atraer e interesar a un nuevo tipo de consumidor más crítico y formado en medios y contenidos que participa de forma interactiva y proactiva en estos procesos de comunicación de las marcas».
\end{abstract}

Se pasa, así de una economía de mercado a una economía de las relaciones. Como ya predijo el Manifiesto Cluetrain al afirmar que «los mercados son conversaciones» ${ }^{10}$, los medios sociales permiten a las empresas participar en conversaciones y aportar contenido de interés para los usuarios, en un entorno favorable tanto para consumidores como para marcas. Apunta Madinabeitia (2010: 49) que:

\begin{abstract}
«Ahora la mayor parte de los especialistas reconocen que tan importante como el papel persuasor de la publicidad es su capacidad de 'generar conversaciones'. El consumidor ocupa ahora, de verdad, el centro de todo el sistema. Este hecho ha implicado un cambio radical en la comunicación comercial».
\end{abstract}

La consolidación de las redes sociales como modelo de comunicación masiva ha hecho que plataformas como Facebook crezcan cada día en usuarios de manera exponencial. Según datos de ComScore, a finales de 2010 hay más de 945 millones de usuarios de redes sociales en el mundo; Facebook es la red social por antonomasia, superando los 600 millones de usuarios.

En España, de acuerdo con la edición de 2011 del estudio anual Navegantes en la Red publicado por la AIMC, las redes sociales son un fenómeno en continuo ascenso: un $60,3 \%$ de la población internauta se conecta a ellas diariamente, frente al 43,5\% que lo hacía en 2009 y el $28,6 \%$ que accedía diariamente en 2008. Facebook es la red social más popular en nuestro país, con más de 15 millones de usuarios, al conectarse a ella el $90 \%$ de los usuarios de redes sociales,

10 Puede consultarse en: http://www.well-comm.es/wellcommunity/wp-content/uploads/cluetrain.pdf. Fecha de consulta 19/11/2011. 
seguida de Tuenti y Twitter. En el caso de esta última, destaca el crecimiento experimentado de 2009 a 2010 , de $12,7 \%$ a $21,9 \%^{11}$.

Las redes sociales son los espacios que ocupan un mayor porcentaje de tiempo de consumo de Internet de los usuarios españoles, según Nielsen Online. El 70\% de internautas fueron usuarios de redes sociales en 2010, frente al $51 \%$ que lo era en 2009, de acuerdo con el II Estudio sobre redes sociales en Internet de IAB Spain y Elogia Ipsofacto ${ }^{12}$.

En el ámbito de la comunicación comercial, varios estudios han demostrado que las marcas se han convertido en un actor más en el tejido de las relaciones: alrededor de la mitad de los usuarios de la Red declaran unirse y seguir actividades de marcas, Facebook es considerado un canal de referencia a la hora de buscar información de una marca y el $16 \%$ de los usuarios de Facebook conecta con nuevos usuarios a través de la interacción con las marcas, el $51 \%$ en el caso de los usuarios de Twitter. Además, al haber pasado a formar parte de una comunidad de marca, los usuarios tienen una visión más positiva de ella, reconocen tener más probabilidad de comprar la marca, se siente más fiel a ella e invitan a otros usuarios a unirse a la comunidad ${ }^{13}$.

Por tanto, los usuarios conversamos sobre marcas en los medios sociales, actividad todavía más intensa si nos fijamos en el público joven, que es quien pasa más tiempo en estas plataformas $\mathrm{y}$, por tanto, quien más recomendaciones, críticas y sugerencias acerca de empresas y productos intercambia, como demuestra el estudio Urban Legends: Word-of-mouth myths, de Colloquy;

En definitiva, la presencia de empresas y marcas en los nuevos entornos digitales es una realidad motivada por las bondades de las plataformas sociales en el ámbito del marketing y la comunicación empresarial, gracias a las posibilidades de orientación empresarial basada en las relaciones (la filosofía del client first), generación de branding social, segmentación y personalización de mensajes, evangelización a través de la prescripción y la viralidad y la puesta en marcha de un experiential marketing que genere customer engagement ${ }^{14}$.

\footnotetext{
11 Estudio disponible en: http://www.aimc.es/-Navegantes-en-la-Red-.html. Fecha de consulta 19/11/2011.

12 Disponible en: http://www.slideshare.net/IAB_Spain/informe-redes-sociales-iab-2010-noviembre-2010?from=ss_ embed. Fecha de consulta 19/11/2011.

13 Resultados de la segunda oleada del estudio «Observatorio de Redes Sociales«, realizado por The Cocktail Analysis, publicado en febrero de 2010 y disponible en http://www.slideshare.net/TCAnalysis/tca-2ola-observatorio-redesinforme-pblicov2-2, y del informe «Wave 5. The socialisation of Brands", de Universal McCann, que puede consultarse en http://www.slideshare.net/Olivier.mermet/universal-mccann-wave-5-the-socialisation-of-brands. Fecha de consulta 19/11/2011.

14 Para una información más detallada sobre estas ventajas, puede consultarse Castelló, 2010: 79-98.
} 
Cuadro 1. Métodos de comunicación empleados para hablar sobre productos. Comparativa entre jóvenes (18-25 años) y la población general estadounidense ${ }^{15}$

\begin{tabular}{|c|c|c|}
\hline & Juseres(6)IS] & 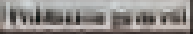 \\
\hline 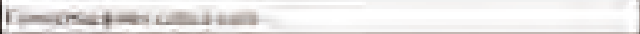 & Th: & 6as. \\
\hline Crmondrobstan & swe & 90 \\
\hline Vrutess. 6 . & was & 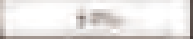 \\
\hline 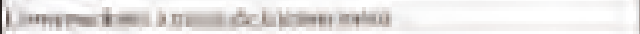 & 74 & vir: \\
\hline Foesulas & $\operatorname{sen}$ & 35. \\
\hline ither & en. & $2=$ \\
\hline 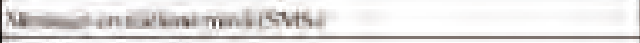 & ans. & 10 \\
\hline Pan eld bingen & ins. & $1 \mathrm{n}$ \\
\hline 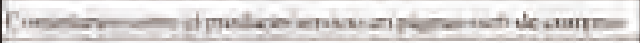 & 16. & int \\
\hline 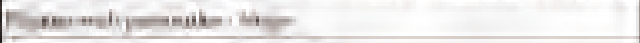 & 1et. & Len. \\
\hline 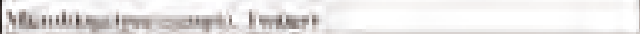 & ts. & $=$ \\
\hline
\end{tabular}

Como destaca el estudio Turning 'like' to 'buy'. Social media emerge as a commerce channel, de Booz \& Company, la integración de los medios sociales en la estrategia de marketing permite aplicar estas plataformas en cada uno de los objetivos $^{16}$ :

\section{Cuadro 2. Actividades de marketing en medios sociales}

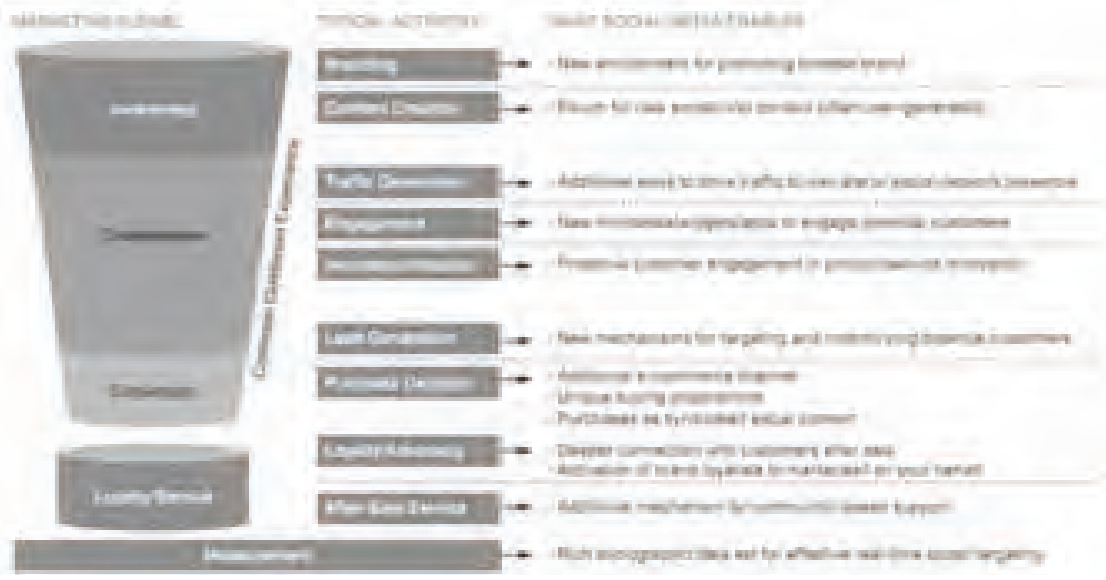

15 Estudio publicado en diciembre de 2010 y disponible en http://www.colloquy.com/files/2011-COLLOQUY-Talk-TalkWhite-Paper.pdf. Fecha de consulta 19/11/2011.

16 Puede consultarse en http://www.booz.com/media/uploads/BaC-Turning_Like_to_Buy.pdf. Fecha de consulta 19/11/2011. 
En las plataformas 2.0, es fundamental que la empresa monitorice lo que se dice de ella en tiempo real, escuche activamente, participe en conversaciones con un lenguaje próximo y cercano, ofrezca actualizaciones frecuentes con contenidos relevantes y de calidad, responda de manera rápida y transmita transparencia y confianza.

Sin embargo, varios estudios demuestran que la respuesta dada por la empresa al usuario es escasa, pese a las potencialidades de estas plataformas, más si cabe cuando se trata de dar respuesta a quejas. De acuerdo con Social Bakers, las páginas de marcas en Facebook no responden al 95\% de los comentarios. El estudio Social Media y Customer Feedback, realizado por Market Tools, destaca que tan sólo un 25\% de las empresas presentes en Facebook afirma responder siempre a las quejas de los clientes en redes sociales; el $17 \%$ reconoce no haber respondido nunca o haberlo hecho muy pocas veces:

\section{Cuadro 3. Frecuencia de respuesta en Facebook y Twitter a comentarios de usuarios}

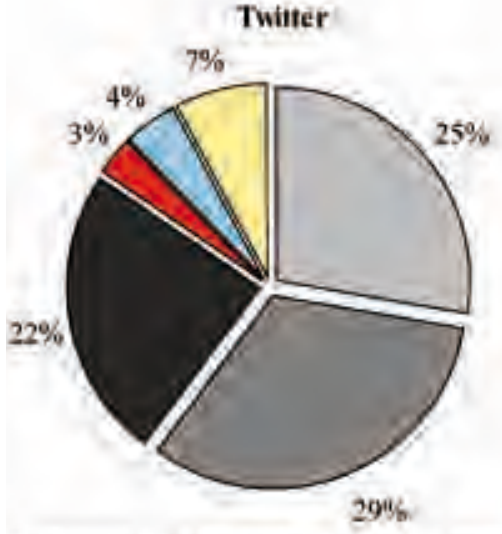

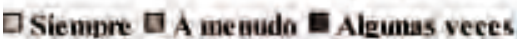

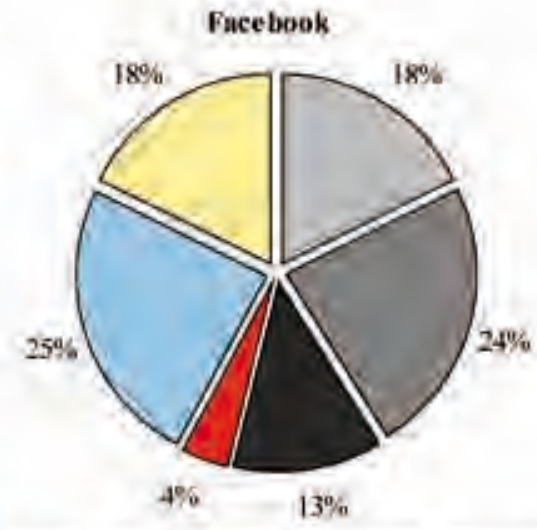

Ranas veces $\square$ Numea $\square$ No sabe/ no contesta

\subsection{El comercio electrónico}

Por comercio electrónico, o e-commerce, se entiende toda compra realizada a través de Internet, cualquiera que sea el medio de pago utilizado. La característica básica del comercio electrónico reside en la orden de compra-venta, la cual tiene que realizarse a través de algún medio electrónico, con independencia del mecanismo de pago efectivo. En función de la naturaleza de los participantes en la transacción económica, existen diferentes modalidades de e-commerce ${ }^{17}$ : 


\section{Cuadro 4. Tipología de e-commerce en función de los participantes}

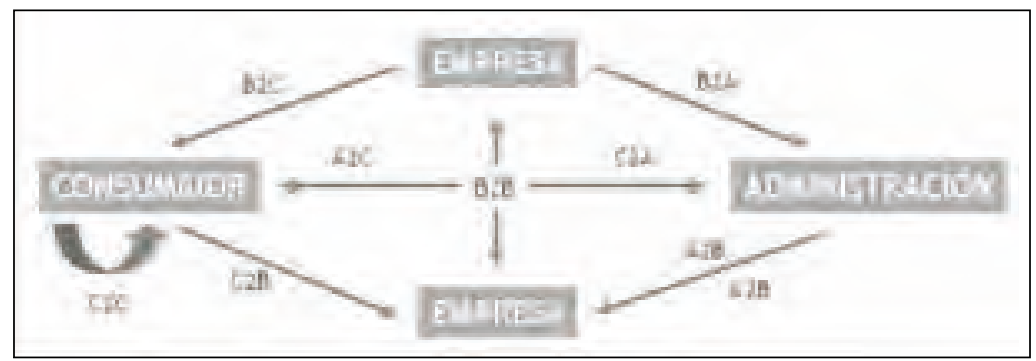

Algunas de las ventajas del comercio electrónico para la empresa son el ahorro en costes derivado de la simplificación de tareas, la flexibilidad y velocidad en los procesos, el alcance global y el acceso directo al consumidor. Por su parte, para el consumidor comprar online supone comodidad de acceso, amplitud de contenidos e información más detallada, ahorro de tiempo, acceso a ofertas, promociones y productos exclusivos y búsqueda y comparativa más precisas, entre otras ventajas.

De acuerdo con el Estudio sobre comercio electrónico b2c $2010^{18}$ de Red.es, el $77,8 \%$ de los internautas que no compraron en 2009 alegaron como principal razón para no comprar las preferencias por las tiendas físicas y por ver lo que compran antes de hacerlo, relacionado también con las connotaciones sociales y de ocio que tiene el acto de compra. La desconfianza que suscita Internet en relación a la mala práctica que se pueda hacer con los datos personales o bancarios es, según este informe, la segunda barrera a la compra online, con un $64,5 \%$.

Según el informe anual La sociedad en Red $2010^{19}$, las ventas B2C alcanzaron en España los 9.100 millones de euros en 2010, un 17,3\% más que el año anterior y, con 10,9 millones de compradores, representaron el 15,5\% del total de compras.

El Informe sobre el comercio electrónico en España a través de entidades de medios de pago de la Comisión del Mercado de las Telecomunicaciones (CMT) ${ }^{20}$ aporta datos del primer trimestre de 2011, con una facturación de 2.055 millones de euros, que representó un aumento interanual del 23,1\%, y un registro total de 30,2 millones de transacciones. Estas cifras revelan los datos más elevados de facturación desde que se empezaran a contabilizar en el año 2000.

18 http://www.red.es/media/registrados/2010-11/1288789343549.pdf?aceptacion=01 c42361992a04cf52e8f53608f47fd6. Fecha de consulta 19/11/2011.

19 Puede consultarse en: http://www.ontsi.red.es/informes-anuales/articles/id/5421/informe-anual-2010-edicion-2011. html. Fecha de consulta 19/11/2011.

20 Disponible en http://www.cmt.es/es/publicaciones/anexos/CE_I_2011_INFORME.pdf. Fecha de consulta 19/11/2011. 
Según la oleada de 2011 del estudio Navegantes en la Red de AIMC ${ }^{21}$, el 76,9\% de los usuarios afirma haber tomado una decisión de compra de productos o servicios orientado, motivado o informado por contenidos de la web. El 8,6\% reconoce haber comprado algún producto o contratado algún servicio a través de Internet en el día de ayer, el $24,2 \%$ en los últimos 7 días y el $34,4 \%$ en el último mes.

En cuanto a la tipología de sitios de comercio electrónico, hay diferentes tipos de venta online y, por lo tanto, distintos comportamientos por parte de los consumidores. Podemos hablar de cuatro tipos: ventas grupales, caracterizadas por los cupones descuento, ventas en outlet de productos exclusivos o de marca, agregadores de ofertas y el comercio electrónico propiamente dicho, con la venta de los mismos productos de la tienda física.

El primer tipo serían plataformas conocidas como de live shopping, portales que publican de forma exclusiva ofertas con importantes descuentos por un periodo de tiempo limitado. Entre este tipo de sitios web destacan Groupon y otras iniciativas como Mequedouno, Offerbox, Oooferton y Lets Bonus. De acuerdo con la empresa de medición Nielsen, más de 7,4 millones de consumidores se acercan mensualmente a estos portales de couponing con el objetivo de encontrar gangas, dada la actual coyuntura económica.

El potencial con que cuentan estas plataformas de e-commerce viene dado también por su asociación con redes sociales de geolocalización, como Foursquare o Facebook Places. De esta manera, los usuarios pueden recibir avisos y alertas en sus dispositivos móviles cuando se encuentran cerca de un establecimiento con una oferta, permitiendo acceder a la compra de forma directa.

De acuerdo con un estudio llevado a cabo por Universal McCann, el 70\% de los usuarios de teléfonos móviles de última generación ha utilizado en alguna ocasión servicios de geolocalización, muchos de los cuales están encaminados a la compra de productos y servicios. En este sentido, los cupones móviles son uno de los servicios más populares entre los usuarios que utilizan sus teléfonos móviles para conectarse a Internet. Además, el 36\% de los usuarios de teléfonos inteligentes los utilizan para aplicaciones relacionadas con las compras y el $66 \%$ reconoce recurrir a ellos para comparar precios de productos mientras se encuentra en el punto de venta. El estudio define a estos consumidores como locales, móviles y sociales (LoMoSos). Sin embargo, los consumidores parecen tener muchas más reservas a la hora de comprar a través del móvil: sólo una tercera parte de los usuarios tiene intención de realizar pagos a través del móvil en el futuro, por la inseguridad de los datos y la falta de control sobre el gasto ${ }^{22}$.

21 Estudio disponible en: http://www.aimc.es/-Navegantes-en-la-Red-.html. Fecha de consulta 19/11/2011.

22 Puede leerse la noticia en http://www.marketingdirecto.com/especiales/marketing-movil/el-futuro-del-shoppinges-local-movil-y-social/?utm_source=wordtwit\&utm_medium=social\&utm_campaign=wordtwit. Fecha de consulta 19/11/2011. 
Los outlets exclusivos de marcas crean su cartera de productos a través de los stocks de grandes marcas y productos exclusivos, principalmente de los sectores moda y belleza, como Privalia, BuyVip o Vente Privee. Existen también portales y buscadores que actúan como intermediarios entre compradores y consumidores, al ser aglutinadores y comparadores de ofertas directas de tiendas online. Algunos ejemplos son Google Shopping, Kelkoo, Misoutlets o Yunait.

Por último, las tiendas online han demostrado ser para las empresas un canal de negocio muy rentable para hacer frente a la crisis económica de principios de siglo XXI. El sector moda es uno de los que más ha crecido, en este sentido. Si en 2008 alrededor de 500.000 consumidores españoles compraban moda en la red, en 2010 la cifra se triplicó, según el informe Internet, la moda en un click, realizado por Kantar Worldpanel, siendo el único canal de compra que consiguió crecer en facturación, a un ritmo del $+15 \%$, cuando el sector arrojó una caída del $-7 \%$.

En definitiva, Internet se expande como canal de venta e influye cada vez más en los hábitos de compra de los usuarios, como demuestra el estudio de actitudes LifeSTYLES $4^{23}$ realizado por Kantar Worldpanel. El crecimiento de la facturación en el comercio electrónico es una realidad y si a esto le sumamos el auge de los medios sociales tenemos como resultado un nuevo concepto, como veremos en el siguiente apartado: el social commerce.

\subsection{El social commerce}

La combinación de medios sociales y comercio electrónico ha dado lugar al social commerce. La filosofía de esta compra social sigue siendo la de las plataformas 2.0: escuchar a los usuarios y conseguir generar interacción por parte de ellos, para fortalecer el vínculo con el consumidor y, de esta manera, crear una oportunidad de negocio y de fidelización del cliente.

Hay que tener también en cuenta que, en general, los usuarios de redes sociales son más activos en Internet que los que no son usuarios, siendo la compra online una de las actividades que realizan con mayor frecuencia que los no usuarios, como demuestra el II Estudio sobre redes sociales en Internet de IAB Spain y Elogia Ipsofacto ${ }^{24}$.

El punto fuerte para la venta online de estos entornos colaborativos es la conversación y recomendación que se genera entre los usuarios a propósito de productos y marcas, para comparar precios, comentar usos, resolver dudas, dar consejos de instalación, etc. Además, la conversación tiene lugar en todas las fases del proceso de compra, por lo que la toma de decisiones en cada fase se socializa, aún más, y los grupos de referencia se amplían con las redes sociales.

23 Estudio disponible en http://www.kantarworldpanel.com/kwp_ftp/Spain/NdP/20101122_desayunos/20101122_ BalanceyFuturoGC_presentacion.pdf. Fecha de consulta 19/11/2011.

24 http://www.iabspain.net/ver.php?mod=noticias\&identificador=80. Fecha de consulta 19/11/2011. 
Ejemplos son las iniciativas Justbought.it, aplicación basada en la geolocalización que permite compartir fotos y tweets a propósito de gangas que el usuario encuentra en el momento, o Lowffer.com, que publica a tiempo real las experiencias de compra de los usuarios registrados, de forma geolocalizada.

Con el social commerce, los usuarios pueden seguir a una marca en una plataforma social al mismo tiempo que acceden a su tienda virtual, incentivando a que el fan se convierta en cliente y, por tanto, incrementando el número de conversiones.

El comercio electrónico a través de Facebook se conoce como f-commerce y hace uso de aplicaciones de tiendas virtual que quedan integradas dentro de la red social (como Payvment), anuncios de los productos en el muro, publicación de catálogos, etc. El caso más conocido en España es la tienda integrada en Facebook de Telepizza, con descuentos exclusivos si se realiza el pedido en este canal. El estudio The effect of social networks and the mobile web on website traffic and the inevitable rise of Facebook commerce ${ }^{25}$, publicado por Webtrends, destaca la eficacia de Facebook como plataforma de comercio debido a sus elevados ratios de conversión, así como para generar tráfico.

Además de la integración de la tienda online en la plataforma social, el social commerce también puede darse a la inversa, integrando los medios sociales en la plataforma de comercio electrónico ya existente de la empresa, como por ejemplo en la tienda virtual de Levi's Friends Store, lo que permite potenciar una mayor difusión de los productos y aporta un valor diferencial a la compra.

El social commerce también ha permitido a pequeñas y medianas empresas contar con un nuevo canal de venta accesible y a su medida que, además, ayuda a generar tráfico hacia el sitio web de la empresa. En este sentido, surgen iniciativas que tratan de agrupar a pequeños comercios para poder competir con grandes compañías, como Ciudadmarket, que cuenta con una tienda de productos por categoría, escapadas, cupones descuento de larga duración y promociones diarias, además de su propia comunidad (CiudadBla), para el intercambio de información entre usuarios y la relación directa con los comercios.

Sin embargo, la compra a través de redes sociales todavía es vista con incertidumbre e inseguridad por parte del usuario, que prefiere acudir a la página web oficial de la empresa para hacer la compra por cuestiones de seguridad y privacidad, como demuestra la investigación realizada por Havas Media y Social Social Lightspeed: el 65\% de los encuestados en este estudio también afirmó que no estaría dispuesto a comprar artículos de alto valor a través de redes sociales. Por parte de la empresa el social commerce también se encuentra en fase introductoria: tan sólo el $12 \%$ de las principales empresas minoristas que

25 Disponible en: http://www.adgregate.com/Whitepaper/Webtrends-Adgregate_Social_Commerce_Whitepaper_03172011. pdf. Fecha de consulta 19/11/2011. 
operan a través de Internet ofrece aplicaciones que permiten hacer transacciones desde la red social ${ }^{26}$.

La principal bondad que ofrece el social commerce a los usuarios es la posibilidad de compartir sus experiencias de compra y consumo y socializar la compra online. Sin embargo, ofertas y descuentos se han convertido hoy en día en una constante para todos los negocios. Es la información que la empresa puede recolectar sobre el usuario en las plataformas 2.0 la que va a permitirle crear ofertas personalizadas y promociones que realmente respondan a las necesidades del usuario.

El punto diferencial del social commerce está, por tanto, en cómo gestionar las relaciones con los clientes actuales y potenciales ya que, como hemos visto, gracias a las nuevas tecnologías, el individuo es el protagonista. De ahí que se hable en medios sociales del Impact on Relationship (IOR), como parámetro para medir el retorno de la inversión (ROI) que complementa los criterios cuantitativos con otros cualitativos, basados en la autoridad, la influencia, la participación y la interacción.

En definitiva, las posibilidades de interactuar con los comercios y compartir la compra con una comunidad de consumidores en los medios sociales convierten el proceso de compra y consumo en una experiencia, en la que la influencia social, la prescripción y la viralidad juegan un papel fundamental y son los factores de los que van a depender la eficacia de las acciones comerciales y de comunicación empresarial en medios sociales, la fidelización del cliente y, en última instancia, el éxito empresarial.

\subsection{Los portales de descuentos y cupones}

La búsqueda de oportunidades y buenos precios en la Red ha disparado en 2011 la audiencia de portales de descuentos y cupones, que ha crecido en un $64 \%$ según datos de la división online de Nielsen en España. En total más de 7,4 millones de consumidores se acercan mensualmente a estos portales con el objetivo de encontrar gangas.

El modelo de funcionamiento de las plataformas de compras colectivas se basa, fundamentalmente, en una oferta atractiva y limitada en el tiempo, cuyo descuento suele oscilar entre el $50 \%$ y el $90 \%$ del precio oficial. Además, la implantación de estos portales es de ámbito local, ya que las ofertas están referidas a un ámbito geográfico cercano y segmentadas por ciudades y la compra se concreta mediante la emisión de un cupón descuento que debe canjearse presencialmente en el negocio.

Para formalizarse la compra, debe cubrirse un mínimo de compradores, en un plazo que suele ser de 24 horas. De ahí la importancia de la viralización a través

26 Extraído del informe desarrollado por la compañía Ability Commerce. 
de medios sociales para que el descuento se haga efectivo. Por este motivo los portales fomentan la difusión de los descuentos recompensando económicamente a los usuarios por compartirlos y conseguir que sus contactos también los compren. El plazo de un día que debe pasar para obtener el cupón y el mínimo de compradores que debe adquirir la oferta hacen que la transacción sea diferida, es decir, el proceso de compra y venta no es simultáneo. Las ganancias de la operación se reparten entre la plataforma y el anunciante, según la comisión establecida.

Las plataformas de compras colectivas brindan al pequeño negocio diversas ventajas como canal de promoción y ventas:

- no necesita contar con un desarrollo web propio, ya que utiliza la infraestructura online de un tercero y consigue visibilidad a través de ella.

- cuentan a priori con una audiencia numerosa y predispuesta, ya que son usuarios que previamente han dado de manera expresa su consentimiento a recibir diariamente correos electrónicos con información sobre las ofertas.

- el retorno de la inversión es inmediato: en 24 horas se sabe si la iniciativa ha tenido éxito.

- ayuda a generar tráfico hacia el establecimiento, ya que los cupones deben canjearse in situ.

- permite dar salida a stocks y productos de final de temporada o baja cuota de ventas.

El 68\% de los negocios españoles que han lanzado una oferta a través de una web de compras colectivas asegura que la acción fue rentable, según el I Estudio Compra Colectiva Satisfacción de los Negocios presentado por Yunait. Es más: un 76\% afirma que consiguieron fidelizar clientes, y el 78\% asegura que volvería a lanzar una oferta grupal. Los establecimientos de salud y belleza, centros educativos, hoteles, deportes y restaurantes son, según el estudio, los que mejor rentabilizaron la promoción ${ }^{27}$.

\section{Resultados}

En el cuadro 5 hemos incluido los resultados del análisis de los doce case studies de portales de descuentos y cupones presentes en Facebook. En total, se han contabilizado 1.284 publicaciones en los once días del trabajo de campo (del 10 al 20 de noviembre de 2011), lo que supone una media de 116 publicaciones diarias y 9,7 publicaciones al día para cada portal estudiado.

De los doce portales seleccionados tan sólo uno no cuenta con presencia en Facebook (Offerbox). Además, seis de los once que tienen presencia en la red

27 Estudio disponible en: http://www.yunait.com/recursos/estudios/estudio_compra_colectiva_negocios.pdf. Fecha de consulta 19/11/2011. 
social (55\%) no tienen página específica para la provincia de Alicante (Offerbox, Mequedouno, Oooferton, Quebombazo, Cuponing y Cupoon), pese a que la mayoría sí segmenta las ofertas por provincia en la página web. En estos casos se ha analizado la página genérica para el ámbito nacional.

De acuerdo con el ranking de Alexa, los portales más populares en usuarios únicos y visitas son Letsbonus, Groupalia y Groupon. Por su parte, las páginas de Facebook más populares son aquellas que no están segmentadas por provincia, como Oooferton (con 167.390 «Me gusta») y Ofertix (con 64.264 fans). Curiosamente, estas páginas con más seguidores no coinciden con las que tienen más tráfico de acuerdo con Alexa.

Los once portales que tienen espacio en Facebook cuentan en su página web con el icono de la red social para que el usuario enlace con la página del portal en la plataforma, así como con la opción de recomendar o compartir cada uno de los descuentos en diferentes plataformas (Facebook, Twitter o por correo electrónico).

\section{Cuadro 5. Resultados del análisis de case studies}

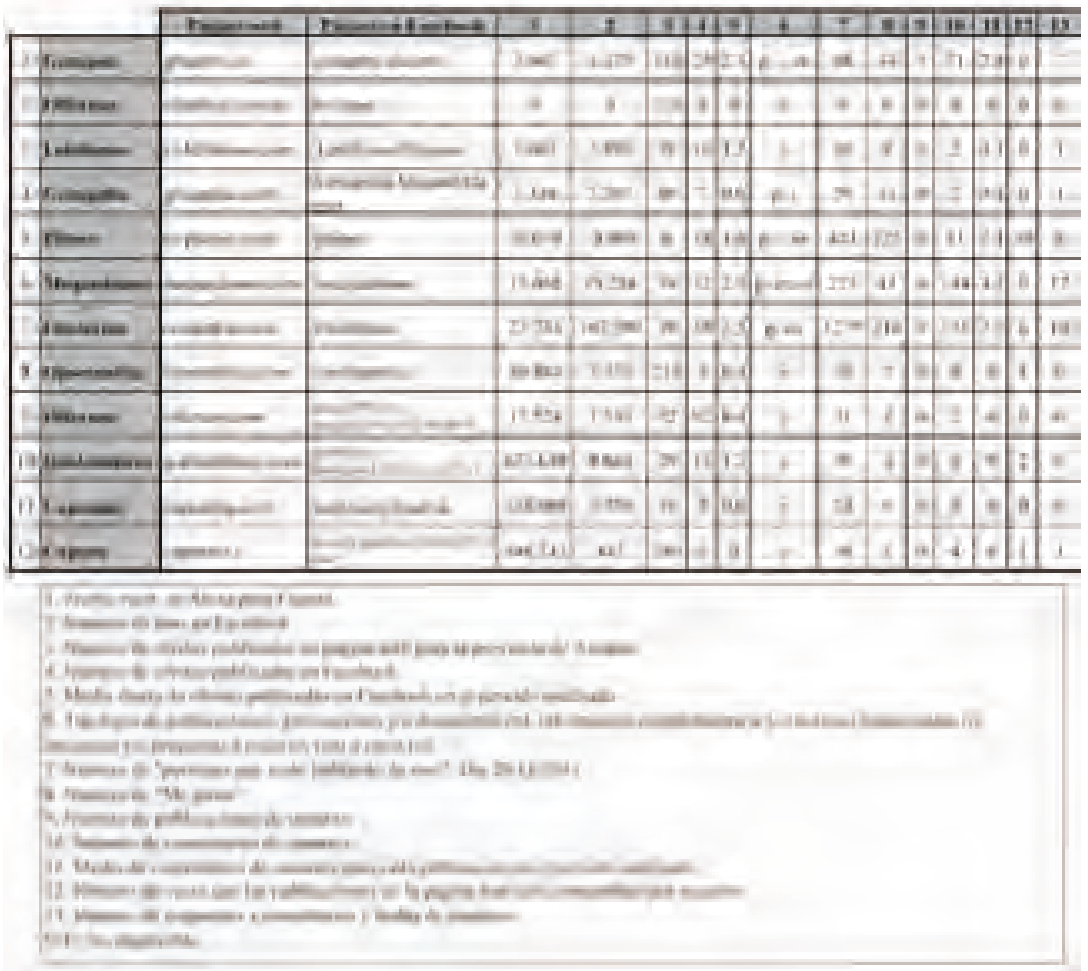


Con respecto al número de ofertas publicadas en Facebook, tan sólo Planeo publica más ofertas en Facebook que en la página web, tal vez por la popularidad que tiene en la red social, puesto que es el tercer portal de los analizados con más seguidores en Facebook. En el caso de Offerum, las ofertas publicadas en la página web y en Facebook coinciden puesto que probablemente así lo tengan configurado, de manera que se publique en la red social automáticamente lo que se publica en el sitio. Groupalia, Oportunista, Cuponing y Cupoon no llegan a publicar una oferta en la media diaria del periodo de once días analizado pese a que, excepto en el caso de Groupalia Alicante, se trata de espacios de ámbito nacional.

Offerum, Oooferton y Mequedouno son los portales que publican más descuentos en Facebook en el periodo analizado, si bien de estos tres portales sólo Offerum, el portal que publica más ofertas de toda la muestra analizada, cuenta con presencia en la red social específica para la provincia de Alicante, por lo que las ofertas de Oooferton y Mequedouno son de ámbito nacional. Los portales que publican otras cosas además de las ofertas y descuentos son Groupon, Groupalia, Planeo y Mequedouno, Oooferton, es decir, el $42 \%$ de la muestra. Curioso es el caso de Cupoon, que cuenta en el periodo analizado con una única publicación, aunque no se trata de una oferta propiamente dicha, sino de una captura de pantalla de la página web, informando de manera genérica de que existen nuevos cupones descuentos disponibles.

Los resultados de las publicaciones de los portales analizados indican que Oooferton, Planeo y Mequedouno son los espacios más populares en base al número de «personas que están hablando de esto». Las páginas con las que más interactúa el usuario si nos fijamos en el número de «me gusta» son Oooferton (316) y Planeo (125). Si calculamos el ratio de «me gusta» para cada fan de las páginas analizadas, tan sólo Cupoon supera el $1 \%$, al ser la página que cuenta con menos fans (443). Le sigue Planeo, que con 14.969 fans y 125 «me gusta» tendría una media de 0,84 «me gusta» por fan. En la media de «me gusta» por ofertas publicadas, Oooferton es el que obtiene el valor más alto: de media, cada oferta publicada obtiene 8,3 «me gusta», seguido por Planeo, con 6,9; el resto de páginas analizadas oscilan entre 0,5 y 1,5 «me gusta» para cada publicación.

En el caso de Offerum, pese a que es el portal que publica más ofertas en Facebook, con el mismo número de ofertas publicadas que en su página web, en los once días analizados es el que menos «me gusta» recibe de sus seguidores (tan sólo cuatro), por lo que tiene el ratio más bajo de «me gusta» para cada oferta publicada.

En relación a las publicaciones realizadas por los usuarios en los espacios de los portales en Facebook, únicamente en Groupon el usuario publica contenido por iniciativa propia, y en los tres casos se trata de dudas o quejas de los usuarios a propósito de algún descuento o producto promocionado. En los tres casos Groupon responde y gestiona las quejas y dudas de los usuarios informando de 
que va a hacer las gestiones oportunas para solventar los problemas o atender a las sugerencias de los usuarios. En este sentido, cabe precisar que no todos los portales analizados permiten al usuario publicar contenido en sus espacios: así lo hacen cuatro de los once portales (Letsbonus, Planeo, Offerum y Quebombazo), lo que supone el $36 \%$ de la muestra.

Mequedouno, Oooferton y Groupon son las páginas con más comentarios de usuarios, con 144, 133 y 71 comentarios, respectivamente. Offerum, página que más ofertas publica en Facebook en el periodo analizado, es uno de los que cuenta con menos comentarios de usuarios (tan sólo dos). Oportunista, Quebombazo y Cuponing no reciben ningún comentario de usuarios a sus publicaciones en los once días analizados.

Si calculamos la media de comentarios por ofertas publicadas, Mequedouno y Oooferton son los que tienen el ratio más alto, con 4,5 y 3,5 comentarios por publicación, respectivamente. El total de comentarios recibidos representa el 1,62\% del número de fans para la página Groupon, siendo así la que obtiene este dato más elevado.

Mequedouno, el portal que cuenta con más comentarios de usuarios en su página de Facebook (144), es también el que más respuestas da (17). Las respuestas de la página representan el mayor porcentaje con respecto a los comentarios de usuarios (50\%) en los espacios de LetsBonus y Groupalia, si bien ambas tienen únicamente dos comentarios de usuarios en cada caso.

En referencia a otros datos y contenidos interesantes, además del muro y de álbumes de fotos, cinco portales cuentan con página de bienvenida personalizada en la red social (45\%), Groupon incluye una pestaña con ofertas de empleo en la empresa y Groupalia cuenta con la aplicación Tu negocio en Groupalia. Oportunista y Letsbonus incluyen también pestañas dedicadas a la aplicación para el teléfono móvil, contando también el primero con una pestaña con la actividad en Twitter y otra con datos de contacto. Oooferton incluye una pestaña dedicada a las condiciones de uso de la página en Facebook y otra con los nombres de los miembros de la empresa («nuestro equipo»). Por último, únicamente Cuponing Madrid cuenta con tienda en Facebook (f-commerce).

Groupon y Groupalia son ejemplo de espacios en los que se publica contenido adicional, de interés para el usuario, más allá de ofertas y descuentos. En el caso del primero, publican contenidos de su blog, sin estar vinculados a ninguna promoción (consejos para combatir el insomnio, trucos contra el frío, cómo evitar el estrés, etc.), así como concursos, como por ejemplo: «estamos buscando los descuentos que más os han gustado durante el mes de octubre. ¿Nos ayudas a encontrarlos? Entre todos los que votéis regalaremos dos escapadas para dos personas. ¡Vota por tus preferidos!».

El espacio de Mequedouno publica avances de la oferta de mañana que invitan al usuario a acertar de qué producto o servicio se tratará, con frases del tipo 
«Mañana... nunca más viajarás solo», a lo que los usuarios responden con sus predicciones: un GPS, una maleta, una cámara de fotos, un DVD portátil, etc. El portal también llevó a cabo un concurso con motivo de su segundo aniversario en noviembre de 2011 que animaba a los usuarios a encontrar en ofertas a $0,02 €$.

Oooferton es el único portal de los analizados que hacen uso de la herramienta encuestas de Facebook, para hacer a los usuarios preguntas del tipo "¿el inglés es tu asignatura pendiente?». Groupon, Planeo y Mequedouno también hacen preguntas a usuarios, aunque sin esta aplicación, a través de sus publicaciones: «Hemos encontrado un artículo que analiza a los tipos de seriéfilos según sus gustos, en Planeo somos un batiburrillo de todos ellos ¿Y tú a qué grupo perteneces?».

A grandes rasgos, podemos destacar el espacio en Facebook del portal Oooferton, por ser el que tiene más fans, suma un alto número de ofertas publicadas en Facebook, complementa las ofertas con preguntas y encuestas a usuarios, es el espacio del que más se está hablando, el que cuenta con más «me gusta» y el segundo con más comentarios de usuarios.

En general, podemos decir que los usuarios sí interactúan con cierta frecuencia con aquellos contenidos publicados por los portales en Facebook que dan pie a conversación con el usuario, como aquellos que emplean preguntas retóricas, un lenguaje coloquial y un tono cercano en la comunicación (un claro ejemplo de esto es Letsbonus), si bien estos portales de descuentos no continúan esa conversación de manera acertada con el usuario, como lo demuestra el bajo ratio de respuestas con respecto a comentarios que obtienen la mayoría de los portales. Pinchar en «me gusta» y comentarios son las acciones que más realizan los usuarios al interactuar con las publicaciones: las 1.284 publicaciones contabilizadas han generado 568 «me gusta» y 401 comentarios entre los 237.265 fans que siguen a los portales analizados en Facebook. La opción de compartir con sus contactos no es tan popular, ya que de las 252 ofertas publicadas contabilizadas en el análisis, tan sólo 40 han sido compartidas por usuarios.

\section{Conclusiones}

Como hemos estudiado a lo largo de este artículo, los medios sociales se han convertido en un canal de venta en el que la empresa busca lograr conversión, fidelidad y recomendación. La creación y el establecimiento de un vínculo con el usuario (engagement), a través de aspectos como los que hemos analizado, a saber, la generación de contenidos relevantes y de valor y la interacción con el usuario, son claves para el éxito en las plataformas sociales. Este vínculo es el que favorece un win-to-win: consigue fidelizar a los clientes, convirtiéndoles en prescriptores de la marca, y ayuda a la empresa a detectar los clientes más 
rentables, comprender los gustos y necesidades de los clientes y así mejorar continuamente los productos para satisfacerle.

Escuchar y participar en conversaciones en tiempo real ayudará a la compañía a desarrollar experiencias más personalizadas para el consumidor, mejorará la efectividad comercial y comunicacional de la empresa, reduciendo gastos operativos de atención al cliente y generará nuevas oportunidades de negocio.

Sin embargo, las particularidades de las plataformas de compras colectivas, basadas en la publicación masiva de descuentos en un periodo de tiempo reducido y la aglutinación de promociones de otras empresas, hacen que se olvide esta filosofía 2.0 que inevitablemente debe imperar en cualquier iniciativa de venta online que quiera hacer uso de los medios sociales, por lo que podemos decir que se cumple la hipótesis de partida de este estudio: si bien estos portales complementan con frecuencia la información sobre las ofertas, descuentos y promociones que publican en la red social con otros contenidos (noticias de interés, concursos, encuestas y preguntas a usuarios, etc.) y emplean un lenguaje cercano, en general cuando el usuario continúa la conversación aportan poca retroalimentación a los seguidores.

Para reforzar la relación entre los miembros de la comunidad que representan las plataformas de compras colectivas, estos portales deben ser capaces de coordinar desde el punto de vista comunicacional los esfuerzos de las empresas cuyos descuentos ofrecen e involucrarlas en el empleo de espacios como Facebook como canal de interacción con el usuario y difusión de ofertas, favoreciendo la generación de contenidos centrados en el destinatario, coherentes, organizados y desarrollados en base a una estrategia común.

\section{Referencias bibliográficas}

Castelló Martínez, Araceli (2010). Estrategias empresariales en la Web 2.0. Las redes sociales online. Alicante: Editorial ECU.

Bermejo Berros, Jesús (2008). El receptor publicitario del siglo XXI en el marco de la interactividad: entre el consumer y el prosumer. En: Pacheco Rueda, Marta (Coord.). La publicidad en el contexto digital. Sevilla: Comunicación Social.

Bryant, Jennings y Miron, Dorina (2004). Theory and Research in Mass Communication. En: Journal of Communication, vol. 54, $\mathrm{n}^{0} 4$.

Madinabeitia, Eduardo (2010). La publicidad en medios interactivos. En busca de nuevas estrategias. En: Telos, $\mathrm{n}^{\circ}$ 82. Madrid: Fundación Telefónica, 43-54.

http://sociedadinformacion.fundacion.telefonica.com/DYC/TELOS/SOBRETELOS/Nmerosanteriores/DetalleAnteriores_82TELOS_DOSSIER2/ seccion $=1268 \&$ idioma $=$ es_ES \&id $=2010020211580001 \&$ activo=6.do. Fecha de consulta 01/06/2011. 
Marshall, David (2004). New Media Cultures. Londres: Arnold Publishers.

Martí Parreño, José (2006). Los contenidos publicitarios y el nuevo consumidor de medios digitales: del consumer al prosumer. Disponible en

http:/www.cibersociedad.net/congres2006/gts/comunicacio.php?id=259\&llengua=es. Consultado el 19/05/2011.

Scolari, Carlos (2008). Hipermediaciones. Elementos para una teoría de la comunicación digital interactiva. Barcelona: Gedisa Editorial.

\section{Referencia de este artículo:}

Castelló Martínez, Araceli (2012). La comunicación de los portales de descuentos y cupones a través de Facebook. En: adComunica. Revista Científica de Estrategias, Tendencias e Innovación en Comunicación, $\mathrm{n}^{\circ} 3$. Castellón: Asociación para el Desarrollo de la Comunicación adComunica, Universidad Complutense de Madrid y Universitat Jaume I, 65-86. DOI: http://dx.doi.org/10.6035/21740992.2012.3.5 